\title{
MMSE Receiver for Multicarrier CDMA Overlay in Ultra-Wide-Band Communications
}

\author{
Wong Tat Tung and Jiangzhou Wang, Senior Member, IEEE
}

\begin{abstract}
Multicarrier direct-sequence code-division multiple-access (MC-CDMA) overlay has been proposed to be used for ultra-wide-band (UWB) communications. Interference reduction and interference suppression are the key issues for sharing the spectrum in harmony between the established narrow-band systems and the overlaid UWB system. In this paper, investigation is carried out on the use of compromising measures incorporated to the MC-CDMA overlay to meet these goals, as well as on their impacts to the involving parties. At the transmitter, interference reduction to the established narrow-band systems is done by using notch filters. A multipath Nakagami fading channel is assumed. At the receiver, the interference suppression from those narrow-band systems is fulfilled by minimum mean square error (MMSE) detection technique. Numerical results show that precombining MMSE with selective-maximal combining provides the UWB system with much better performance than the receiver made up of notch filter in cascade with code correlator.
\end{abstract}

Index Terms-Minimum mean square error (MMSE) adaptive rake, multicarrier code-division multiple-access (CDMA), Nakagami fading, notch filter, ultra-wide-band communications.

\section{INTRODUCTION}

I $\mathrm{N}$ THE foreseeable future, a blooming growth in the demand is expected for the information sharing and data distribution tools and facilities in hot-spot layer as well as personal network layer. The development of short-range and high-speed transmission systems is going to play a significant role in the area of wireless communications. This has motivated the exploration of the ultra-wide-band (UWB) transmission system. The key of the UWB system is that the emission bandwidth of the devices can be up to gigahertz, within the frequency band of 3.1-10.6 $\mathrm{GHz}$ [1]. To meet the goal of efficient use of scarce spectrum resources, the devices can operate using those spectra occupied by existing radio services without causing interference. The UWB system with low-emission-power would probably operate on an unlicensed basis.

Multicarrier direct-sequence code-division multiple-access (MC-CDMA) has been proposed to be used in UWB communications [2]. Under this modulation scheme, the data signal is spread by a higher rate random code and the resultant is then modulated on multiple carriers. MC-CDMA offers the following advantages.

First, the ultra-wide bandwidth is sliced into a number of wide bandwidth portions. The rate of signal processing is no

Manuscript received June 23, 2003; revised June 3, 2004; October 3, 2004. The review of this paper was coordinated by Prof. T. Lok.

The authors are with the Department of Electrical and Electronic Engineering, University of Hong Kong, Hong Kong, China (ttwong@eee.hku.hk; jwang@eee.hku.hk).

Digital Object Identifier 10.1109/TVT.2004.841551 longer directly related to the entire occupied spectra, so lower processing speed units can be used.

Second, the signal spectra of different carriers can be disjoint from each other. The subbands can also be shifted to appropriate frequency regions. With the reinforcement of bandlimiting techniques, important spectra such as industrial, scientific, and medical (ISM) bands or for emergency uses can be easily avoided.

Third, resource allocation can be flexible. Instead of transmitting a replica of signal in all subbands, tradeoff between overall transmission rate and quality of service from frequency diversity is allowed. In addition, decades' effort and experiences have proved that CDMA is an efficient way for wireless communication as well as a suitable candidate for overlaying purposes [3], [4]. Various techniques developed for CDMA signal processing can be applied.

To share the resource harmoniously, the idea of notch filtering at transmitter was outlined in [4]. Notches are placed on appropriate frequency location as illustrated in Fig. 1. The emission power of the UWB system is attenuated over the occupied spectra by established systems to fulfill the interference reduction requirement. Since the established services are in fact narrow-band systems, the impact of the notches to the overall spreading gain of the overlaying CDMA system is limited. In this paper, the notch filtering on the chip shaping is considered. The notch action is implemented by a transversal filter where the tap weights are adapted to suppress the targeted spectral regions. For fixed resource assignment of narrow-band systems, the tap weights adaptation can be based on either available information in advance, or observation of the captured signal.

At the receiving end, the UWB signal is affected by narrow-band interference and frequency selective fading. Although CDMA enables this fine time resolution benefits to be deeply explored, the emission power of UWB system is extremely low in comparison to those existing systems. It is desirable to employ a mechanism that is capable for jointly gathering the multipath energy and suppressing narrow-band interference. Here, precombining minimum mean square error (MMSE) receiver [5] with an intrinsic selective maximal combination (SMC) [6], [7] is considered for the suggested receiver design.

The organization of the rest of this paper is as follows. In Sections II and III, a completed analysis is provided to illustrate the significance of the suggested design framework for the use of MC-CDMA for UWB communication as well as its impacts on those existing narrow-band users. The closed-form expression for the bit error rate (BER) can be found in Section IV. Section $\mathrm{V}$ provides a performance comparison between the MMSE receiver and the one built up of notch filter in cascade with code 


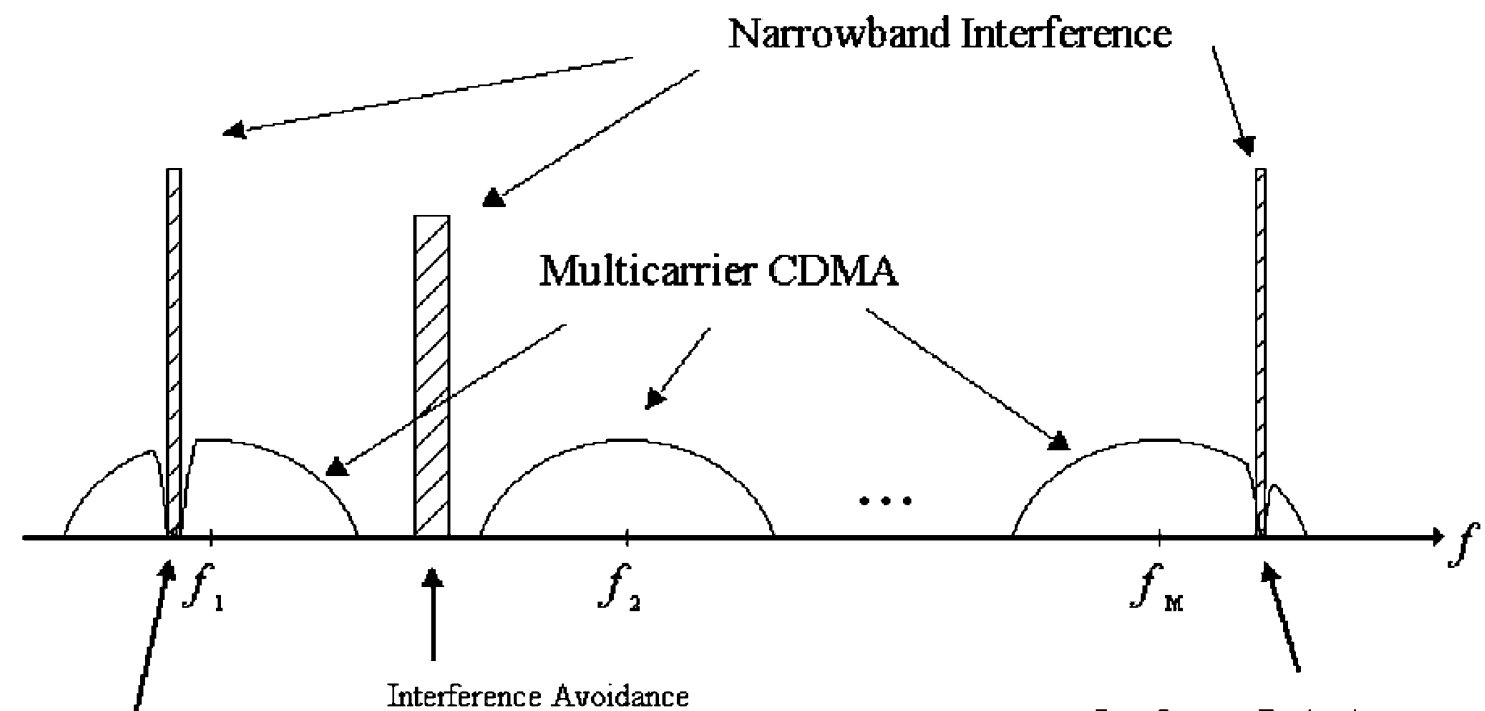

Interference Reduction

Interference Reduction

Fig. 1. Power spectrum densities of the UWB signal and the narrow-band signals (shaded).

correlator (NCC receiver) as suggested in [2]. Section VI gives the conclusions.

\section{SySTEM MODEL}

\section{A. Transmitter}

The MC-CDMA system is assumed to contain $M$ subcarriers and $K$ independent active users. $N$ and $T_{c}$ are the processing gain of each subchannel and the chip duration of the CDMA system, respectively. The block diagram of the transmitter for the $k$ th user is shown in Fig. 2(a). $c_{k}^{(n)}$ is the $n$th element of the random sequence, and it takes the value $1 / \sqrt{N}$ or $-1 / \sqrt{N}$ with equal probability. $b_{k}^{\lfloor n / N\rfloor}$ is the $\lfloor n / N\rfloor^{t h}$ element of the binary data sequence, where $\lfloor x\rfloor$ stands for the integer part of $x$. The product of the two sequences results in the spreading signal of the $k^{\text {th }}$ user, given by

$$
d_{k}^{(n)}=b_{k}^{\left\lfloor\frac{n}{N}\right\rfloor} c_{k}^{(n)} .
$$

The idea of using notch filter at transmitter to notch out frequency bands occupied by narrow-band systems has been outlined in [4]. In this study, a low complexity scheme is suggested in which the notch action is achieved by the chip shaping modification of a two-sided transversal-type filter. The resultant chip shaping thus can achieve bandlimiting and interference avoidance to the established service simultaneously. In the proposed transmitter, the spreading signal $d_{k}^{(n)}$ passes through the chipshaping filter to give the baseband signal before frequency up conversion. As a derivative of conventional MC-CDMA transmitter, independent chip shaping filters should be used for the modulators of different subcarriers. This is because interference avoidance requirement is distinct for each subband. The chip shaping filter is shown in Fig. 2(b). Its impulse response is given by

$$
h_{T X}^{(m)}(t)=F^{-1}\left\{H_{T X}^{(m)}(f)\right\}=F^{-1}\left\{H_{T X}(f) H_{W}^{(m)}(f)\right\}
$$

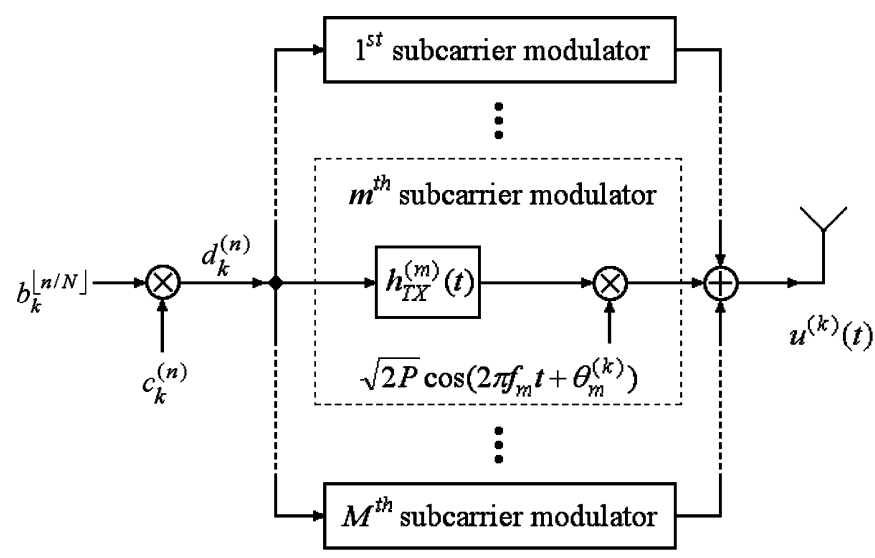

(a)

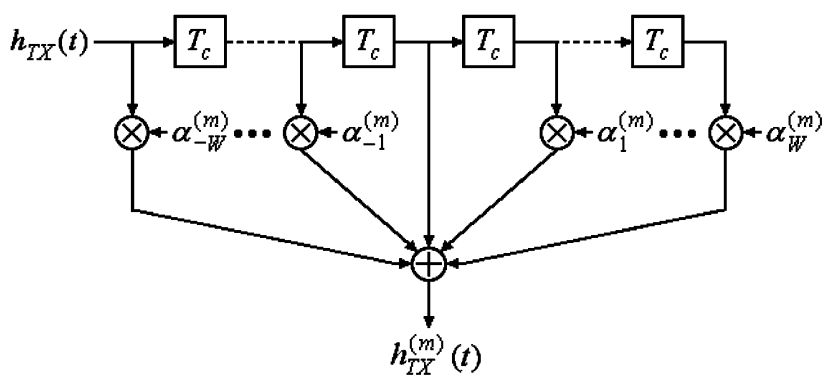

(b)

Fig. 2. (a) Transmitter for the $k$ th user of the MC-DS-CDMA UWB system. (b) The notch filter of the chip shape for the $k$ th user for the $m$ th subcarrier.

where $H_{W}^{(m)}(f)$ is the frequency response of the two-sided transversal-type notch filter with $2 W+1$ taps for the $m$ th subcarrier, given by

$$
H_{W}^{(m)}(f)=\sum_{w=-W}^{W} \alpha_{w}^{(m)} \exp \left(-j 2 \pi f w T_{c}\right) .
$$

The tap coefficients are adapted to suppress the spectra with narrow-band systems. However, a dynamic scheme is required 
since there is apparent impossibility of coordination among the coexisting systems. Any tap-coefficient adjustment should be driven by the existence of the narrow-band system. In this paper, the tap weights are assumed to be determined based on the observation at the receiver, and achieved their optimal under sufficient training [8]. The present value of the narrow-band process can be predicted from past value and future value. This is not applicable for UWB signal and the channel noise as they are wide-band processes. Therefore, the estimation of the location of the narrow-band system can be done by observation of the past and future values of the received signal samples.

When there is no narrow-band interference, no notch is inserted and the filter reduces to an all-pass filter, i.e., $H_{W}^{(m)}(f)=$ 1 . Then the undistorted raised-cosine chip shape is transmitted, as if there is no notch filter. $H_{T X}(f)$ in (2) stands for the frequency response of the unfiltered chip-shaped filter at transmitter. Suppose $H_{R X}(f)$ represents the frequency response of the unmodified chip-matched filter at receiver; these frequency responses are related by

$$
H_{R C}(f)=H_{T X}(f) H_{R X}(f)
$$

where $H_{R C}(f)$ denotes the frequency response of the raised-cosine chip shape [9] with rolloff factor $\alpha$. Thus, the bandlimiting transmitted signal of the $k$ th user can be written as

where

$$
u^{(k)}(t)=\sqrt{2 P} \sum_{m=1}^{M}\left(u_{m}^{(k)}(t) \cos \left(2 \pi f_{m} t+\theta_{m}^{(k)}\right)\right)
$$

$$
u_{m}^{(k)}(t)=\sqrt{2 P} \sum_{n=-\infty}^{\infty} d_{k}^{(n)} h_{T X}^{(m)}\left(t-n T_{c}\right) .
$$

In (5), $P$ is the transmit power per subcarrier, and $f_{m}$ and $\theta_{m}^{(k)}$ are the carrier frequency and random phase of the $m$ th subcarrier.

\section{B. Channel and Narrow-Band System}

The channel model of the UWB communications is characterized by a rich number of multiple propagation paths. With reference to the stochastic tapped-delay-line model of UWB indoor application suggested in [10], channel variation is assumed to be slow so that the parametes are time-invariant over a bit period. The subbands are assumed to be disjoint, and their channel response is assumed to be uncorrelated. The impulse response of the $m$ th subband channel for the $k$ th user can be expressed as

$$
h_{m}^{(k)}(t)=\operatorname{Re}\left[\sum_{l=1}^{L} \beta_{m, l}^{(k)} \delta\left(t-\tau_{m, l}^{(k)}\right) \exp \left(j \phi_{m, l}^{(k)}\right)\right]
$$

where $L$ is the number of resolvable paths for each carrier of the UWB system, with the assumption of slow fading. $\phi_{m, l}^{(k)}, \tau_{m, l}^{(k)}$, and $\beta_{m, l}^{(k)}$ denote the phase shift, delay, and attenuation factor of the lth path, respectively. These sets of channel variables are assumed to be mutually independent. The resolvable paths of the same subcarrier signal from the same user are assumed to be time-separated by chip duration such that

$$
\tau_{m, l}^{(k)}=\tau_{m}^{(k)}+(l-1) T_{c}
$$

where $\tau_{m}^{(k)}$ is the random delay uniformly distributed over $\left[0, T_{b}\right)$. The phase shifts are identical and uniformly distributed over $[0, \pi)$. The attenuation factors are assumed to be identical and Nakagami distributed. The probability density function attenuation factor is given by [11]

$$
\begin{aligned}
p\left(\beta_{l, m}^{(k)}\right)=\frac{2\left(\beta_{l, m}^{(k)}\right)^{2 \mu_{l, m}^{(k)}-1}}{\Gamma\left(\mu_{l, m}^{(k)}\right)} & \left(\frac{\mu_{l, m}^{(k)}}{\Omega_{l, m}^{(k)}}\right)^{\mu_{l, m}^{(k)}} \\
& \times \exp \left(-\frac{\mu_{l, m}^{(k)}}{\Omega_{l, m}^{(k)}}\left(\beta_{l, m}^{(k)}\right)^{2}\right)
\end{aligned}
$$

where $\mu_{l, m}^{(k)}$ denotes the fading figure, given by

$$
\mu_{l, m}^{(k)}=\frac{\left(\Omega_{l, m}^{(k)}\right)^{2}}{E\left[\left(\beta_{l, m}^{(k)}\right)^{2}-\left(\Omega_{l, m}^{(k)}\right)^{2}\right]}
$$

and $\Omega_{l, m}^{(k)}$ is the normalized power-decay profile (PDP) which is the second moment of the attenuation. The normalized PDP $\Omega_{l, m}^{(k)}$ is related to the PDP of the initial path of the same user $\Omega_{1, m}^{(k)}$ via [10]

$$
\Omega_{l, m}^{(k)}=\Omega_{1, m}^{(k)} v \exp \left(-\frac{(l-2)}{\delta}\right) .
$$

And $\Omega_{1, m}^{(k)}$ can be expressed as

$$
\Omega_{1, m}^{(k)}=\frac{1-\exp \left(\frac{-T_{c}}{\delta}\right)}{1-\exp \left(-\frac{T_{c}}{\delta}\right)+v\left[1-\exp \left(-\frac{(L-1) T_{c}}{\delta}\right)\right]}
$$

where $\delta$ is the decay rate and $v$ is the power ratio of the second PDP to that of direct path (which is assumed to be the strongest). The PDPs decay exponentially with delay starting from the second bin. Note that the normalization implies that $\sum_{l=1}^{L} \Omega_{l, m}^{(k)}=1$.

The established narrow-band signals along the operating spectrum of the UWB system are assumed to be bandlimited Gaussian processes with flat power spectrum. For the sake of simplicity, single path propagation is assumed for the narrow-band system, and one narrow-band system is assumed in any given subband. The collection of established narrow-band systems along the operating spectrum of the UWB system can be represented by

$$
j(t)=\sum_{m=1}^{M} j_{m}(t)
$$

where $j_{m}(t)$ denotes the narrow-band system within the $m$ the subband and its power spectrum can be expressed as

$$
P_{j}^{(m)}(f)= \begin{cases}\frac{J_{o}}{2}, & 0 \leq\left|f-\frac{q_{m}}{T_{c}}\right| \leq \frac{p_{m}}{2 T_{c}} \\ 0, & \text { otherwise }\end{cases}
$$

where $J_{o}$ is the power spectral density of the narrow-band system within the spectral region of the $m^{\text {th }}$ subband. In (14), $p_{m}$ is defined as the ratio of the narrow-band system bandwidth to the UWB system subband bandwidth, such that $0 p_{m} 1 . q_{m}$ is defined as the ratio of the offset of the center frequency of the 


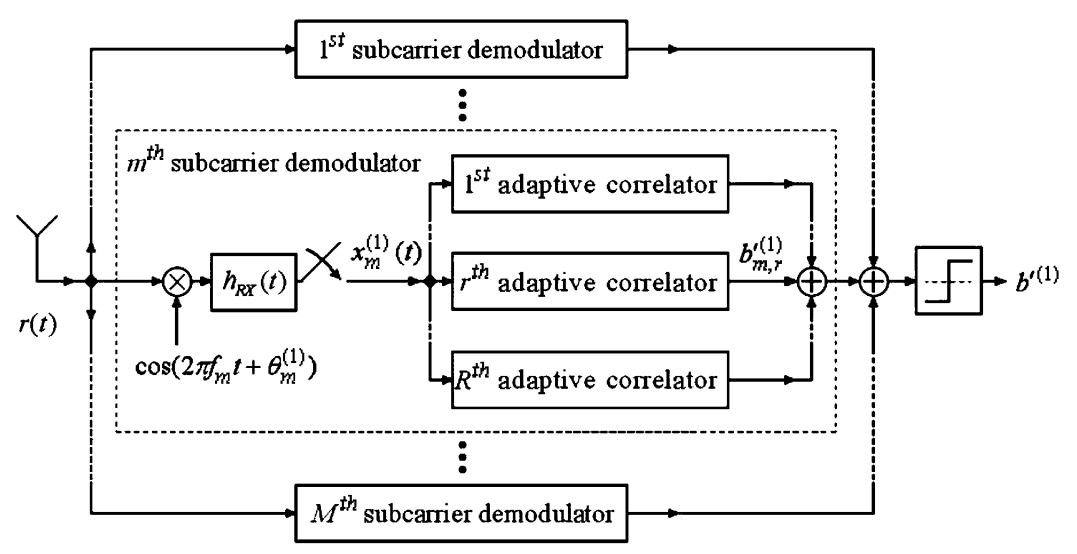

(a)

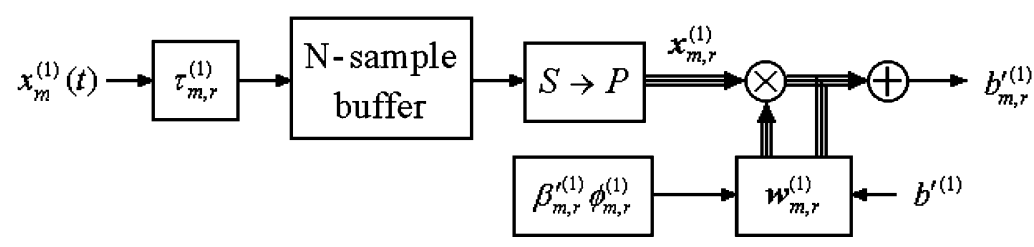

(b)

Fig. 3. (a) Receiver for the first user of the MC-DS-CDMA UWB system and (b) the $r$ th adaptive correlator of the first user for the $m$ th subcarrier.

narrow-band system signal from the $m$ th subcarrier's center frequency of the UWB system to the bandwidth of the subband, such that $-0.5(1+\alpha) q_{m} 0.5(1+\alpha)$.

\section{Receiver}

The received signal can be expressed as

$$
\begin{aligned}
& r(t)=\sum_{k=1}^{K} \sum_{m=1}^{M} \sqrt{2 P} u_{m}^{(k)}(t) \cos (\left.2 \pi f_{m} t+\theta_{m}^{(k)}\right) \\
& * h_{m}^{(k)}(t)+j(t)+n(t)
\end{aligned}
$$

where* stands for convolution. The block diagram of the UWB receiver is shown in Fig. 3(a). The receiver consists of $M$ parallel branches corresponding to each subband. Each branch consists of rake receiver with $R$ correlators. For the sake of convenience, the first user is taken as the reference user. The received signal undergoes frequency down conversion, chip-matched filtering, and bandpass filtering. The impulse response of the recovered baseband signal from the $m$ th subcarrier for the first user can be written as

$x_{m}^{(1)}(t)=\int_{0}^{T_{c}} r(t+\lambda) \cdot \cos \left(2 \pi f_{m}(t+\lambda)+\theta_{m}^{(1)}\right) \cdot h_{R X}^{(m)}(\lambda) d \lambda$

where $h_{R X}^{(m)}(t)$ is the impulse response of the chip-matched filter for the $m$ th subband. Perfect carrier recovery is assumed. The chip-matched filter is time-synchronized with one of the paths for the targeted user, i.e., the first arrival path. The recovered baseband signal includes multiple-access system signals in the subband, in-band noise, and possibly narrow-band interference. These components are mutually independent from each another. $x_{m}^{(1)}(t)$ is then sampled at chip rate. For example, the starting time and the ending time of the integration of the $n^{\text {th }}$ chip are $\tau_{m}^{(1)}+n T_{c}$ and $\tau_{m}^{(1)}+(n+1) T_{c}$, respectively. The discrete- time samples of the recovered baseband signal are passed to the estimation circuitry for notch filter coefficients estimation and the Rake receiver for data recovery.

1) Tap Weights of the Transversal-Type Notch Filter: The estimator of notch filter coefficients at receiver should have similar structure to the functional block of the notch filter at transmitter illustrated in Fig. 2(b). From [8], the filter coefficients for the $m$ th subcarrier can be written

$$
\begin{aligned}
\boldsymbol{\alpha}_{0}^{(m)} & =1 \\
\boldsymbol{\alpha}^{(m)} & =\left[\begin{array}{llllll}
\alpha_{-W}^{(m)} & \cdots & \alpha_{-1}^{(m)} & \alpha_{1}^{(m)} & \cdots & \alpha_{W}^{(m)}
\end{array}\right]^{T} \\
& =\operatorname{Re}\left\{\boldsymbol{Q}_{m}^{(1)^{-1}} \boldsymbol{P}_{m}^{(1)}\right\} .
\end{aligned}
$$

$\boldsymbol{P}_{m}^{(1)}$ and $\boldsymbol{Q}_{m}^{(1)}$ are a $2 W \times 1$-array and $2 W \times 2 W$ matrix, respectively, defined as

$$
\begin{aligned}
& \boldsymbol{P}_{m}^{(1)}=E\left[x_{m}^{(1)}\left(t_{o}\right) \boldsymbol{X}_{m}^{(1)}\right] \\
& \boldsymbol{Q}_{m}^{(1)}=E\left[\boldsymbol{X}_{m}^{(1)} \boldsymbol{X}_{m}^{(1)^{T}}\right]
\end{aligned}
$$

where $t_{o}$ is an arbitrary time reference. $\mathbf{X}_{m}^{(1)}$ is a $2 W \times 1$-array of past $W$ samples and future $W$ samples from the chip matched filtered output of the $m^{\text {th }}$ subcarrier of the first user, which can be expressed as

$$
\begin{array}{rll}
\boldsymbol{X}_{m}^{(1)}{ }^{T}=\left[\begin{array}{lll}
x_{m}^{(1)}\left(t_{o}+W T_{c}\right) & \cdots & x_{m}^{(1)}\left(t_{o}+T_{c}\right) \\
x_{m}^{(1)}\left(t_{o}-T_{c}\right) & \cdots & x_{m}^{(1)}\left(t_{o}-W T_{c}\right)
\end{array}\right] .
\end{array}
$$

Let $W_{L}$ be a $1 \times 2 W$ array representing the location index of the filter taps such that

$$
\boldsymbol{W}_{\boldsymbol{L}}=\left[\begin{array}{llllll}
W & \cdots & 1 & -1 & \cdots & -W
\end{array}\right] .
$$

The contributions from the UWB-signal, narrow-band interference (NBI), and additive white Gaussian noise (AWGN) are independent, and the UWB multiple access signals and the noise 
signal have zero cross-correlation in time. The $w$ th element of $\boldsymbol{P}_{m}^{(1)}$ can be written as

$$
\begin{aligned}
\boldsymbol{P}_{m}^{(1)}(w)= & E\left[x_{m}^{(1)}\left(t_{o}\right) \boldsymbol{X}_{m}^{(1)^{T}}(w)\right\rfloor \\
= & E\left[x_{m}^{(1)}(0) x_{m}^{(1)}\left(\boldsymbol{W}_{L}(w)\right)\right] \\
= & \operatorname{Re}\left(E \left[\int_{-\infty}^{\infty} P_{j}^{(m)}(f) H_{R C}(f)\right.\right. \\
& \left.\left.\quad \times \exp \left(j 2 \pi f \boldsymbol{W}_{L}(w) T_{c}\right) d f\right]\right) .
\end{aligned}
$$

Similarly, the element at the $w_{1}$ th row and $w_{2}^{\text {th }}$ column of $\boldsymbol{Q}_{m}^{(1)}$ can be written as

$$
\begin{aligned}
& \boldsymbol{Q}_{m}^{(1)}\left(w_{1}, w_{2}\right) \\
& =E\left[\boldsymbol{X}_{m}^{(1)}\left(w_{1}\right) \boldsymbol{X}_{m}^{(1)}{ }^{T}\left(w_{2}\right)\right] \\
& =E\left[x_{m}^{(1)}\left(\boldsymbol{W}_{L}\left(w_{1}\right)\right) x_{m}^{(1)}\left(\boldsymbol{W}_{L}\left(w_{2}\right)\right)\right] \\
& =\operatorname{Re}\left(E \left[\int_{-\infty}^{\infty} P_{j}^{(m)}(f) H_{R C}(f)\right.\right. \\
& \left.\left.\quad \times \exp \left(j 2 \pi f\left[\boldsymbol{W}_{L}\left(w_{1}\right)-\boldsymbol{W}_{L}\left(w_{2}\right)\right] T_{c}\right) d f\right]\right) \\
& +\delta\left(\boldsymbol{W}_{L}\left(w_{1}\right)-\boldsymbol{W}_{L}\left(w_{2}\right)\right) \\
& \times \operatorname{Re}\left(\frac { P } { N } \int _ { - \infty } ^ { \infty } \left(\sum _ { L = 1 } ^ { L } E \left[\exp ^{2}\left(j\left(\left.\Delta \phi_{m, l}^{(1)}\right|_{m, r} ^{(1)}\right)\right)\right.\right.\right. \\
& \quad+\sum_{k=2 l=1}^{K} \sum_{m, l}^{L} H_{R C}^{2}(f)\left(\exp ^{2}\left(j\left(\Delta \phi_{m, l}^{(m)}(f)\right)^{2}\right]\right. \\
& \left.\left.\quad-\left.2 \pi f \Delta \tau_{m, r}^{(k)}(k)\right|_{m, r} ^{(1)} \Omega_{m, l}^{(k)}\right)\right) \\
& \quad+\delta\left(\boldsymbol{W}_{L}\left(w_{1}\right)-W_{L}\left(w_{2}\right)\right) \\
& \left.\left.\quad \times \operatorname{Re}\left(\frac{\eta_{o}}{4} E\left[\int_{-\infty}^{\infty} H_{R C}(f) d f\right]\right)\left(H_{R C}^{2}(f)\left(H_{W}^{(m)}(f)\right)^{2}\right]\right) d f\right)
\end{aligned}
$$

where $\delta(x)$ stands for Dirac delta function. $\left.\Delta \phi_{m, l}^{(k)}\right|_{m, r} ^{(1)}$ denotes the phase difference between the $l$ th path of the $k$ th user and the $r$ th path of the first user, which is uniformly distributed over $[0, \pi)$ such that

$$
\Delta \phi_{m, l}^{(k)} l_{m, r}^{(1)}=\phi_{m, 1}^{(k)}+\theta_{m}^{(k)}-\phi_{m, r}^{(1)}-\theta_{m}^{(1)} .
$$

$\left.\Delta \tau_{m, l}^{(k)}\right|_{m, r} ^{(1)}$ denotes the relative delay of the $l$ th path of the $k$ th user with respect to the $r$ th path of the first user, which is uniformly distributed over $\left[0, T_{c}\right)$, given by

$$
\left.\Delta \tau_{m, l}^{(k)}\right|_{m, r} ^{(1)}=\left(\tau_{m, l}^{(k)}-\tau_{m, r}^{(1)}\right)-\left\lfloor\frac{\left(\tau_{m, l}^{(k)}-\tau_{m, r}^{(1)}\right)}{T_{c}}\right\rfloor \cdot T_{c} .
$$

2) Rake Structure for Data Recovery: In each subband, the R-highest $(R \leq L)$ power paths out of all resolvable paths are selected for decision making. Consider the $m$ th subcarrier of the first user, the batch of samples over one bit period to be processed by the $r^{t h}$ correlator can be expressed in an $N \times 1$ array form as

$$
\begin{aligned}
& \boldsymbol{x}_{m, r}^{(1)}=\left[x_{m}^{(1)}\left(\tau_{m, r}^{(1)}+T_{c}\right) \quad \cdots \quad x_{m}^{(1)}\left(\tau_{m, r}^{(1)}+n T_{c}\right)\right. \\
& \left.\cdots \quad x_{m}^{(1)}\left(\tau_{m, r}^{(1)}+N T_{c}\right)\right]^{T} \\
& =\boldsymbol{s}_{m, r}^{(1)}+\boldsymbol{i}_{m, r}^{(1)}+\boldsymbol{j}_{m, r}^{(1)}+\boldsymbol{n}_{m, r}^{(1)} \text {. }
\end{aligned}
$$

$\boldsymbol{s}_{m, r}^{(1)}$ is an array of samples from the first user's $r$ th propagation path and its $n$th element is given by

$$
\begin{array}{r}
\boldsymbol{s}_{m, r}^{(1)}(n)=\sqrt{2 P} \int_{0}^{T_{c}} \beta_{m, l}^{(1)} u_{m}^{(1)} \\
\left(\tau_{m, r}^{(1)}+n T_{c}-\tau_{m, l}^{(1)}+\lambda\right) \\
\times \cos \left(\Phi_{m, r}^{(1)}\right) h_{R X}^{(m)}(\lambda) d \lambda .
\end{array}
$$

$\boldsymbol{i}_{m, r}^{(1)}$ is an array representing the multipath interference (MPI) and multiple access interference (MAI). The $n$th element of the array can be expressed as

$$
\begin{aligned}
\boldsymbol{i}_{m, r}^{(1)}(n)=\int_{0}^{T_{c}}\{ & \sqrt{2 P} \sum_{\substack{l=1 \\
l \neq r}}^{L} \beta_{m, l}^{(1)} u_{m}^{(1)}\left(\tau_{m, r}^{(1)}+n T_{c}-\tau_{m, l}^{(1)}+\lambda\right) \\
& \times \cos \left(\Phi_{m, l}^{(1)}\right) h_{R X}^{(m)}(\lambda) \\
& +\sqrt{2 P} \sum_{k=2 l=1}^{K} \sum_{m, l}^{L} \beta_{m}^{(k)} u_{m}^{(k)}\left(\tau_{m, r}^{(1)}+n T_{c}-\tau_{l, m}^{(k)}+\lambda\right) \\
& \left.\times \cos \left(\Phi_{m, l}^{(k)}\right) h_{R X}^{(m)}(\lambda)\right\} d \lambda
\end{aligned}
$$

where $\Phi_{m, l}^{(k)}$ is equivalent to the effective phase shift due to the random phase introduced at the transmitter and that from channel distortion, i.e., $\Phi_{m, l}^{(k)}=\theta_{m}^{(k)}+\phi_{m, l}^{(k)}$.

$\boldsymbol{j}_{m, r}^{(1)}$ is an array denoting the NBI and its $n$th element takes the form

$$
\begin{aligned}
& j_{m, r}^{(1)}(n)=\int_{0}^{T_{c}} j_{m}\left(\tau_{m, r}^{(1)}+n T_{c}+\lambda\right) \\
& \cdot \cos \left(2 \pi f_{m}\left(\tau_{m, r}^{(1)}+n T_{c}+\lambda\right)+\theta_{m}^{(1)}\right) h_{R X}^{(m)}(\lambda) d \lambda .
\end{aligned}
$$

$\boldsymbol{n}_{m, r}^{(1)}$ is an array of contribution from the channel noise, and its $n$th element can be written as

$$
\begin{aligned}
& \boldsymbol{n}_{m, r}^{(1)}(n)=\int_{0}^{T_{c}} n\left(\tau_{m, r}^{(1)}+n T_{c}+\lambda\right) \\
& \cdot \cos \left(2 \pi f_{m}\left(\tau_{m, r}^{(1)}+n T_{c}+\lambda\right)+\theta_{m}^{(1)}\right) h_{R X}^{(m)}(\lambda) d \lambda .
\end{aligned}
$$

The bolded arrays are mutually independent from each another. In this paper, the comparison of the performance of the MMSE receiver and the receiver of notch filter in cascade with code correlator is provided, and these two receiving strategies are presented in the following section. 


\section{RECEIVING STRATEGIES}

\section{A. Notch Filter in Cascade With Code Correlator (NCC)}

The use of notch filter at receiver for narrow-band interference suppression in addition to the processing gain of the CDMA system has been described in [2] and [8], where the transversal notch filter is inserted between the chip-matched filter and the rake structure. In this paper, the notch filtering is considered to be merged into the chip matching process. It is similar to the modified chip shaping at the transmitting end mentioned in the previous section. Since their taps are adapted by the same information, the notch filter response at the receiver should be the same as that at the transmitter. The impulse response of the modified chip matched filter is given by

$$
h_{R X}^{(m)}(t)=F^{-1}\left\{H_{W}^{(m)}(f) H_{R X}(f)\right\} .
$$

The samples are then correlated with the spreading code. Let $\boldsymbol{c}_{m, r}^{(1)}=\left[c_{1}^{\left(n^{\prime}\right)} \cdots c_{1}^{\left(n^{\prime}+N-1\right)}\right]^{T}$ be the array of the synchronized first user's spreading code for the $r$ th correlator of the $m$ th subcarrier. The decision variable at the output of the correlator can be written as

$$
\hat{b}_{m, r}^{(1)}=\boldsymbol{c}_{m, r}^{(1)}{ }^{T} \boldsymbol{x}_{m, r}^{(1)}
$$

The mean of the decision statistic takes the form

$$
E\left[b_{m, r}^{\prime(1)}\right]_{\mathrm{NCC}}=\sqrt{P} \operatorname{Re}\left\{E\left(\int_{-\infty}^{\infty}\left(H_{W}^{(m)}(f)\right)^{2} H_{R C}(f) d f\right)\right\}
$$

and its variance can be expressed as

$$
\begin{array}{r}
\operatorname{Var}\left[b_{m, r}^{\prime(1)}\right]_{\mathrm{NCC}}=E\left[\boldsymbol { c } _ { m , r } ^ { ( 1 ) ^ { T } } \left(\boldsymbol{I}_{m, r \mathrm{NCC}}^{(1)}+\boldsymbol{J}_{m, r \mathrm{NCC}}^{(1)}\right.\right. \\
\left.\left.+\boldsymbol{N}_{m, r_{\mathrm{NCC}}}^{(1)}\right) \boldsymbol{c}_{m, r}^{(1)}\right] .
\end{array}
$$

The bolded terms $\boldsymbol{I}_{m, r \mathrm{NCC}}^{(1)}, \boldsymbol{J}_{m, r \mathrm{NCC}}^{(1)}$ and $\boldsymbol{N}_{m, r \mathrm{NCC}}^{(1)}$ are mutually independent $N \times N$ matrices and correspond to the autocorrelations of the MPI/MAI, NBI, and AWGN, respectively. Under random code assumption, $\boldsymbol{I}_{m, r \mathrm{NCC}}^{(1)}\left(n_{1}, n_{2}\right)=0$ for $n_{1} \neq n_{2}$. The element on the diagonal $\left(n_{1}=n_{2}\right)$ of matrix $\boldsymbol{I}_{m, r \mathrm{NCC}}^{(1)}$ has the value

$$
\begin{aligned}
& \boldsymbol{I}_{m, r \text { NCC }}^{(1)}\left(n_{1}, n_{2}\right) \\
& =E\left[\boldsymbol{i}_{m, r}^{(1)}\left(n_{1}\right) * \boldsymbol{i}_{m, r}^{(1)}\left(n_{2}\right)\right] \\
& =\operatorname{Re}\left(E \left[\frac{P}{N} \int_{-\infty}^{\infty} \sum_{\substack{l=1 \\
l \neq r}}^{L} \Omega_{m, l}^{(1)} H_{R C}^{2}(f)\left(H_{W}^{(m)}(f)\right)^{4} d f\right.\right. \\
& +\frac{P}{N} \int_{-\infty}^{\infty} \sum_{k=2}^{K} \sum_{l=1}^{L}\left(\Omega_{m, l}^{(k)} \exp \left(j 2 \pi f \Delta \tau_{m, l}^{(k)} l_{m, r}^{(1)}\right)\right. \\
& \left.\left.\left.\cdot H_{R C}^{2}(f)\left(H_{W}^{(m)}(f)\right)^{4}\right) d f\right]\right) .
\end{aligned}
$$

The element at $n_{1}$ th row and $n_{2}$ th column of matrices $\boldsymbol{J}_{m, r_{\mathrm{NCC}}}^{(1)}$ and $\boldsymbol{N}_{m, r_{\mathrm{NCC}}}^{(1)}$ can be expressed as

$$
\begin{aligned}
& \boldsymbol{J}_{m, r \text { NCC }}^{(1)}\left(n_{1}, n_{2}\right) \\
& =E\left[\boldsymbol{j}_{m, r}^{(1)}\left(n_{1}\right) * \boldsymbol{j}_{m, r}^{(1)}\left(n_{2}\right)\right] \\
& =\operatorname{Re}\left(E \left[\int_{-\infty}^{\infty}\left(H_{W}^{(m)}(f)\right)^{2} P_{j}^{(m)}(f) H_{R C}(f)\right.\right. \\
& \left.\left.\times \exp \left(j 2 \pi f\left|n_{1}-n_{2}\right| T_{c}\right) d f\right]\right) \\
& \boldsymbol{N}_{m, r_{\mathrm{NCC}}}^{(1)}\left(n_{1}, n_{2}\right) \\
& =E\left[\boldsymbol{n}_{m, r}^{(1)}\left(n_{1}\right) * \boldsymbol{n}_{m, r}^{(1)}\left(n_{2}\right)\right]
\end{aligned}
$$

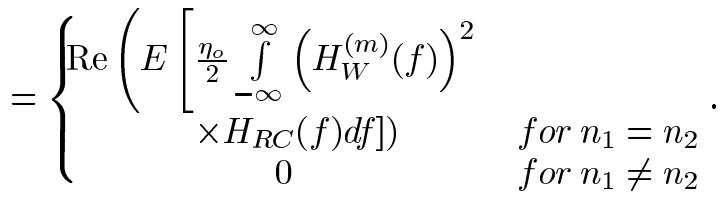

The average signal-to-(interference plus noise) ratio (SINR) of the symbolic decision for the $r$ th finger on the $m$ th subcarrier of the first user after being weighted by the estimated attenuation factor takes the form

$$
\operatorname{SINR}_{\mathrm{NCC}}\left(b_{m, r}^{\prime(1)}\right)=\frac{E^{2}\left[b_{m, r}^{\prime(1)}\right]}{\operatorname{Var}\left[b_{m, r}^{\prime(1)}\right]}=\left(\beta_{m, r}^{(1)}\right)^{2} \zeta_{m, r \mathrm{NCC}}^{(1)}
$$

where the average SINR $\zeta_{m, r}^{(1)}{ }_{\mathrm{NCC}}$, excluding the attenuation factor $\beta_{m, r}^{(1)}$, can be expressed as

$$
\begin{aligned}
& \zeta_{m, r_{\mathrm{NCC}}}^{(1)} \\
& =\frac{P\left[E\left(\int_{-\infty}^{\infty}\left(H_{W}^{(m)}(f)\right)^{2} H_{R C}(f) d f\right)\right]^{2}}{\frac{1}{N} \sum \operatorname{diag}\left\{E\left[\left(\boldsymbol{I}_{m, r_{\mathrm{NCC}}}^{(1)}+\boldsymbol{J}_{m, r_{\mathrm{NCC}}}^{(1)}+\boldsymbol{N}_{m, r \mathrm{NCC}}^{(1)}\right)\right]\right\}}
\end{aligned}
$$

where $\sum \operatorname{diag}(\boldsymbol{x})$ stands for the sum of the diagonal elements of matrix $\boldsymbol{x}$.

\section{B. MMSE Adaptive Correlator}

According to [5] and [12], the adaptive rake filtering makes joint exploration of the path diversity in casual CDMA system as well as the suppression of interference by the single user linear MMSE filtering. The precombining approach allows rapid adaptation to the channel condition. The structure of the MMSE adaptive correlator is shown in Fig. 3(b). The casual chip-matching is applied, and the impulse response of the chip matched filter is given by

$$
h_{R X}^{(m)}(t)=F^{-1}\left\{H_{R X}(f)\right\} .
$$

The decision variable of the $r$ th finger on the $m$ th subcarrier of the desired user is given by

$$
b_{m, r}^{(1)}=\boldsymbol{w}_{m, r}^{(1)}{ }^{T} \boldsymbol{x}_{m, r}^{(1)}
$$


where $\boldsymbol{w}_{m, r}^{(1)}$ represents the $1 \times N$ array of tap weights of the MMSE filter given by

$$
\boldsymbol{w}_{m, r}^{(1)}=\left[\begin{array}{lll}
w_{m, r}^{(1)}(1) & \cdots & w_{m, r}^{(1)}(N)
\end{array}\right]^{T}
$$

The tap weights are determined by minimizing the meansquared error between the correlator output with the product of the estimated attenuation factor $\beta_{m, r}^{\prime(1)}$ power and the feedback estimated data symbol $b^{(1)}$, which is equivalent to [5], [12]

$$
\mathrm{MSE}=E\left[\left|\boldsymbol{w}_{m, r}^{(1)}{ }^{T} \boldsymbol{x}_{m, r}^{(1)}-\beta_{m, r}^{\prime(1)} \sqrt{P} b^{\prime(1)}\right|^{2}\right]
$$

To minimize (43), by Wiener's solution, the tap weight array can be expressed as

$$
\boldsymbol{w}_{m, r}^{(1)}=\beta_{m, r}^{\prime(1)} \sqrt{P}\left(\boldsymbol{x}_{m, r}^{(1)} \boldsymbol{x}_{m, r}^{(1)}\right)^{T} \boldsymbol{c}_{m, r}^{(1)} .
$$

The channel estimation and the feedback estimated data symbol provide the steering force to adapt the spreading code. Perfect channel estimation is assumed, i.e., $\beta_{m, r}^{(1)}=\beta_{m, r}^{(1)}$. The decision statistic consists of the contribution from the desired signal, MPI, MAI of the UWB system, narrow-band interference, and noise. They are assumed to be independent of each other. The mean and the variance of the decision variable of the $r$ th finger on the $m$ th subcarrier of the desired user, conditioned on the attenuation factor, are given by [12] (45) and (46) shown at the bottom of the page. Similar to the analysis of the NCC receiver, $\boldsymbol{I}_{m, r_{\mathrm{MMSE}}}^{(1)}, \boldsymbol{J}_{m, r_{\mathrm{MMSE}}}^{(1)}$, and $\boldsymbol{N}_{m, r_{\mathrm{MMSE}}^{(1)}}$ are independent $N \times N$ matrices due to the autocorrelation function of the signals corresponding to MPI/MAI, NBI, and channel noise, respectively. Under random code assumption, the element at the $n_{1}$ th row and $n_{2}$ th column of matrix $I_{m, r_{\mathrm{MMSE}}}^{(1)}$ can be written as shown in (47) at the bottom of the page. The element at $n_{1}$ th row and $n_{2}$ th column of matrices $\boldsymbol{J}_{m, r_{\mathrm{MMSE}}}^{(1)}$ and $\boldsymbol{N}_{m, r_{\mathrm{MMSE}}}^{(1)}$ can be expressed as

$$
\begin{aligned}
& \boldsymbol{J}_{m, r \text { MMSE }}^{(1)}\left(n_{1}, n_{2}\right) \\
& =E\left[\boldsymbol{j}_{m, r}^{(1)}\left(n_{1}\right) * \boldsymbol{j}_{m, r}^{(1)}\left(n_{2}\right)\right] \\
& =\operatorname{Re}\left(E \left[\int_{-\infty}^{\infty} P_{j}^{(m)}(f) H_{R C}(f)\right.\right. \\
& \left.\left.\times \exp \left(j 2 \pi f\left|n_{1}-n_{2}\right| T_{c}\right) d f\right]\right) \\
& \left.\boldsymbol{N}_{m, r_{\operatorname{MMSE}}^{(1)}\left(n_{1}, n_{2}\right)}=\sum_{m, r}^{(1)}\left(n_{1}\right) * \boldsymbol{n}_{m, r}^{(1)}\left(n_{2}\right)\right] \\
& =\left\{\begin{array}{cc}
\operatorname{Re}\left(E\left[\frac{\eta_{o}}{2} \int_{-\infty}^{\infty} H_{R C}(f) d f\right]\right) & \text { for } n_{1}=n_{2} . \\
0 & \text { for } n_{1} \neq n_{2}
\end{array}\right.
\end{aligned}
$$

The SINR of the symbolic decision for the $r$ th finger on the $m$ th subcarrier of the first user $b_{m, r}^{\prime(1)}$ is

$$
\operatorname{SINR}_{\text {MMSE }}\left(b_{m, r}^{\prime(1)}\right)=\frac{E^{2}\left[b_{m, r}^{\prime(1)}\right]}{\operatorname{Var}\left[b_{m, r}^{\prime(1)}\right]}=\left(\beta_{m, r}^{(1)}\right)^{2} \zeta_{m, r_{\mathrm{MMSE}}}^{(1)}
$$

where the average SINR $\zeta_{m, r}^{(1)}$ MMSE excluding the attenuation factor $\beta_{m, r}^{(1)}$ can be expressed as shown in (51) at the bottom of the next page. The outputs of the correlators have been weighted by their own channel attenuation factor inherently. The final estimated data symbol $b^{\prime(1)}$ is determined by the equal gain combination of the outputs from all $R$ branches of the adaptive rake of all $M$ subcarriers, which can be written as

$$
b^{\prime(1)}=\left|\sum_{m=1}^{M} \sum_{r=1}^{R} b_{m, r}^{(1)}\right| \text {. }
$$

$$
\begin{aligned}
E\left[b_{m, r}^{(1)}\right]_{\mathrm{MMSE}} & =\sqrt{P} \beta_{m, r}^{(1)} E\left(\int_{-\infty}^{\infty} H_{W}^{(m)}(f) H_{R C}(f) d f\right) \\
\operatorname{Var}\left[b_{m, r}^{(1)}\right]_{\mathrm{MMSE}} & =E\left[\left|\boldsymbol{w}_{m, r}^{(1)^{T}} \boldsymbol{x}_{m, r}^{(1)}-\beta_{m, r}^{(1)} \sqrt{P} b^{\prime(1)}\right|^{2}\right] \\
& =\frac{1}{E\left[\boldsymbol{c}_{m, r}^{(1)}\left(\boldsymbol{I}_{m, r_{\mathrm{MMSE}}^{(1)}}^{(1)}+\boldsymbol{J}_{m, r \mathrm{MMSE}}^{(1)}+\boldsymbol{N}_{m, r_{\mathrm{MMSE}}}^{(1)}\right)^{-1} \boldsymbol{c}_{m, r}^{(1)}{ }^{T}\right]}
\end{aligned}
$$

$$
\begin{aligned}
\boldsymbol{I}_{m, r_{\mathrm{MMSE}}}^{(1)}\left(n_{1}, n_{2}\right) & =E\left[\boldsymbol{i}_{m, r}^{(1)}\left(n_{1}\right) * \boldsymbol{i}_{m, r}^{(1)}\left(n_{2}\right)\right] \\
& =\left\{\operatorname { R e } \left(E\left[\begin{array}{c}
\frac{P}{N} \int_{-\infty}^{\infty} \sum_{\substack{l=1 \\
l \neq r}}^{L} \Omega_{m, l}^{(1)} H_{R C}^{2}(f)\left(H_{W}^{(m)}(f)\right)^{2} d f \\
+\frac{P}{N} \int_{-\infty}^{\infty} \sum_{k=2}^{K} \sum_{l=1}^{L}\left(\begin{array}{c}
\Omega_{m, l}^{(k)} \exp \left(\left.j 2 \pi f \Delta \tau_{m, l}^{(k)}\right|_{m, r} ^{(1)}\right) \\
\cdot H_{R C}^{2}(f)\left(H_{W}^{(m)}(f)\right)^{2} \\
0,
\end{array}\right) d f
\end{array}\right), \text { for } n_{1}=n_{2}\right.\right.
\end{aligned}
$$




\section{PROBABILITY OF ERROR}

The contributions from only the significant paths are considered under the path selective approach. In total, $M \cdot R$ contributions are added together to provide the overall symbolic decision. It is shown that the average SINR of the decision statistic is separable in terms of $\beta_{m, r}^{(1)}$ and an independent component $\gamma_{m, r}^{(1)}$, and therefore the conditional average bit error probability can be written as

$$
\begin{aligned}
P_{e} & =\int_{0}^{\infty} Q\left(\sqrt{\zeta_{1}}\right) p\left(\zeta_{1}\right) d \zeta_{1} \\
& =\underbrace{\iint \ldots \int}_{M \cdot \text { Rfolds }} Q\left(\sqrt{\sum_{m=1}^{M} \sum_{r=1}^{R}\left(\beta_{m, r}^{(1)}\right)^{2} \gamma_{m, r}^{(1)}}\right) \\
& \times p\left(\beta_{1,1}^{(1)}, \beta_{1,2}^{(1)}, \ldots, \beta_{M, R}^{(1)}\right) d \beta_{1,1}^{(1)} d \beta_{1,2}^{(1)} \ldots d \beta_{M, R}^{(1)}
\end{aligned}
$$

where $Q(x)$ is the Q-function and $p\left(\beta_{1,1}^{(1)}, \beta_{1,2}^{(1)}, \ldots, \beta_{M, R}^{(1)}\right)$ is the joint probability density function (pdf) of the attenuation factors of the selected multipaths among the subbands. The path selection is independent among the subbands, and $p\left(\beta_{1,1}^{(1)}, \beta_{1,2}^{(1)}, \ldots, \beta_{M, R}^{(1)}\right)$ can be expressed as the product of $M$ joint pdfs corresponding to each subband, as

$$
p\left(\beta_{1,1}^{(1)}, \beta_{1,2}^{(1)}, \ldots, \beta_{M, R}^{(1)}\right)=\prod_{m=1}^{M} p\left(\beta_{m, 1}^{(1)}, \beta_{m, 2}^{(1)}, \ldots, \beta_{m, R}^{(1)}\right) .
$$

The joint pdf for the $m$ th subcarrier $p\left(\beta_{m, 1}^{(1)}, \beta_{m, 2}^{(1)}, \ldots, \beta_{m, R}^{(1)}\right)$ takes the form [6], [7]

$$
\begin{aligned}
p\left(\beta_{m, 1}^{(1)}, \beta_{m, 2}^{(1)}, \ldots, \beta_{m, R}^{(1)}\right)=\frac{L !}{(L-R) !} & {\left[P\left(\beta_{m, R}^{(1)}\right)\right]^{L-R} } \\
& \times \prod_{l=1}^{R} p\left(\beta_{m, l}^{(1)}\right)
\end{aligned}
$$

where $\beta_{m, 1}^{(1)} \geq \beta_{m, 2}^{(1)} \geq \ldots \geq \beta_{m, R}^{(1)}$ is assumed in the above expression. $P\left(\beta_{m, R}^{(1)}\right)$ is the probability distribution function of the Nakagami variable. The derivative of the probability distribution function is the pdf [9], [14]

$$
\begin{aligned}
P\left(\beta_{m, R}^{(1)}\right) & =\int_{0}^{x} p\left(\beta_{m, R}^{(1)}\right) d x \\
& =\frac{\gamma\left(\mu_{m, R}^{(1)}, \frac{\mu_{m, R}^{(1)}}{\Omega_{m, R}^{(1)}}\left(\beta_{m, R}^{(1)}\right)^{2}\right)}{\Gamma\left(\mu_{m, R}^{(1)}\right)}
\end{aligned}
$$

where $\gamma(a, b)$ and $\Gamma(a)$ are the lower incomplete gamma function and complete gamma function, respectively [15], given by

$$
\begin{aligned}
\gamma(a, b) & =\int_{0}^{b} t^{a-1} e^{-t} d t \\
\Gamma(a) & =\int_{0}^{\infty} x^{a-1} e^{-x} d x .
\end{aligned}
$$

The $M \cdot R$-folds integration of (53) is simplified as shown in the following. Define $\bar{\gamma}_{m}^{(1)}$ as the mean of the average SINR excluding the attenuation factor for all selected paths for the $m$ th subband of the first user, which takes the form

$$
\bar{\gamma}_{m}^{(1)}=\frac{1}{R} \sum_{r=1}^{R} \gamma_{m, r}^{(1)}
$$

The average bit error probability can be rewritten as

$$
\begin{aligned}
P_{e} & =\underbrace{\iint \ldots \int}_{M \cdot R \text { folds }} Q\left(\sqrt{\left.\sum_{m=1}^{M} \sum_{r=1}^{R}\left(\beta_{m, r}^{(1)}\right)^{2} \gamma_{m}^{(1)}\right)}\right. \\
& \times \prod_{m=1}^{M} p\left(\beta_{m, 1}^{(1)}, \beta_{m, 2}^{(1)}, \ldots, \beta_{m, R}^{(1)}\right) d \beta_{1,1}^{(1)} d \beta_{1,2}^{(1)} \ldots d \beta_{M, R}^{(1)} .
\end{aligned}
$$

From [16], the Q-function $Q(x)$ can be expressed as

$$
Q(x)=\frac{1}{\pi} \int_{0}^{\frac{\pi}{2}} \exp \left(-\frac{x^{2}}{2 \sin ^{2}(\Theta)}\right) d \Theta .
$$

Substituting $x=\sqrt{\sum_{m=1}^{M} \sum_{l=1}^{R}\left(\beta_{m, r}^{(1)}\right)^{2} \gamma_{m}^{(1)}}$ into the above equation gives

$$
\begin{aligned}
& Q\left(\sqrt{\sum_{m=1}^{M} \sum_{l=1}^{R}\left(\beta_{m, r}^{(1)}\right)^{2} \gamma_{m}^{(1)}}\right) \\
& =\frac{1}{\pi} \int_{0}^{\frac{\pi}{2}} \exp \left(-\frac{\sum_{m=1}^{M} \sum_{l=1}^{R}\left(\beta_{m, r}^{(1)}\right)^{2} \gamma_{m}^{(1)}}{2 \sin ^{2}(\Theta)}\right) d \Theta \\
& =\frac{1}{\pi} \int_{0}^{\frac{\pi}{2}} \prod_{m=1}^{M} \prod_{l=1}^{R} \exp \left(-\frac{\left(\beta_{m, r}^{(1)}\right)^{2} \gamma_{m}^{(1)}}{2 \sin ^{2}(\Theta)}\right) d \Theta .
\end{aligned}
$$

The order of integration can be changed and an inner decomposition of the error probability equation can be taken place. The integral is converted to a single integration on the product

$$
\begin{aligned}
\zeta_{m, r_{\mathrm{MMSE}}}^{(1)} & =P\left(\int_{-\infty}^{\infty} H_{W}^{(m)}(f) H_{R C}(f) d f\right)^{2} E\left[\boldsymbol{c}_{m, r}^{(1)}\left(\boldsymbol{I}_{m, r_{\mathrm{MMSE}}}^{(1)}+\boldsymbol{J}_{m, r_{\mathrm{MMSE}}}^{(1)}+\boldsymbol{N}_{m, r_{\mathrm{MMSE}}}^{(1)}\right)^{-1} \boldsymbol{c}_{m, r}^{(1)} T^{T}\right] \\
& =P\left(\int_{-\infty}^{\infty} H_{W}^{(m)}(f) H_{R C}(f) d f\right)^{2} \frac{\left\{\sum \operatorname{diag}\left(E\left[\left(\boldsymbol{I}_{m, r_{\mathrm{MMSE}}}^{(1)}+\boldsymbol{J}_{m, r_{\mathrm{MMSE}}}^{(1)}+\boldsymbol{N}_{m, r}^{(1)}{ }_{\mathrm{MMSE}}\right)^{-1}\right]\right)\right\}}{N} .
\end{aligned}
$$


of $M$ independent (subcarrier) $R$-fold (multipath) integration, yielding

$$
\begin{aligned}
& P_{e}=\frac{1}{\pi} \int_{0}^{\frac{\pi}{2}}\left\{\prod _ { m = 1 } ^ { M } \left[\int_{0}^{\infty} \ldots \int_{\beta_{m, r}^{(1)}}^{\infty} \ldots\right.\right. \\
& \int_{\beta_{m, 2}^{(1)}}^{\infty}\left(\prod_{r=1}^{R} \exp \left(-\frac{\left(\beta_{m, r}^{(k)}\right)^{2} \gamma_{m}^{(1)}}{2 \sin ^{2}(\Theta)}\right)\right. \\
& \left.\times p\left(\beta_{m, 1}^{(1)}, \beta_{m, 2}^{(1)}, \ldots, \beta_{m, R}^{(1)}\right)\right) \\
& \left.\left.\times d \beta_{m, 1}^{(1)} d \beta_{m, 2}^{(1)} \ldots d \beta_{m, R}^{(1)}\right]\right\} d \Theta \\
& =\frac{1}{\pi} \int_{0}^{\frac{\pi}{2}} \prod_{m=1}^{M}\left\{\frac{L !}{(L-R) !} \int_{0}^{\infty} \ldots \int_{\beta_{m, r}^{(1)}}^{\infty} \ldots\right. \\
& \int_{\beta_{m, 2}^{(1)}}^{\infty}\left[\left(\prod_{r=1}^{R} \exp \left(-\frac{\left(\beta_{m, r}^{(1)}\right)^{2} \gamma_{m}^{(1)}}{2 \sin ^{2}(\Phi)}\right) p\left(\beta_{m, r}^{(1)}\right)\right)\right. \\
& \left.\times\left(P\left(\beta_{m, R}^{(1)}\right)\right)^{L-R}\right] \\
& \left.\times d \beta_{m, 1}^{(1)} d \beta_{m, 2}^{(1)} \ldots d \beta_{m, R}^{(1)}\right\} d \Theta \\
& =\frac{1}{\pi}\left(\frac{L !}{(L-R) !}\right)^{M} \\
& \times \int_{0}^{\frac{\pi}{2}} \prod_{m=1}^{M} R\left(\beta_{m, 1}^{(1)}, \beta_{m, 2}^{(1)}, \ldots, \beta_{m, R}^{(1)}\right) d \Theta
\end{aligned}
$$

where $R\left(\beta_{m, 1}^{(1)}, \beta_{m, 2}^{(1)}, \ldots, \beta_{m, R}^{(1)}\right)$ is the term related to $m$ th subcarrier components expressed as

$$
\begin{aligned}
R & \left(\beta_{m, 1}^{(1)}, \beta_{m, 2}^{(1)}, \ldots, \beta_{m, R}^{(1)}\right) \\
= & \int_{0}^{\infty}\left(P\left(\beta_{m, R}^{(1)}\right)\right)^{L-R} \\
& \times \int^{\infty} \ldots \int_{m, R}^{\infty}\left(\prod_{l=1}^{R} \exp \left(-\frac{\left(\beta_{m, r}^{(1)}\right)^{2} \gamma_{m}^{(1)}}{2 \sin ^{2}(\Theta)}\right) p\left(\beta_{m, r}^{(1)}\right)\right) \\
& \times d \beta_{m, 1}^{(1)} d \beta_{m, 2}^{(1)} \ldots d \beta_{m, R}^{(1)} \\
= & \int_{0}^{\infty}\left(P\left(\beta_{m, R}^{(1)}\right)\right)^{L-R} R^{\prime}\left(\beta_{m, R}^{(1)}\right) \\
& \times \int_{\beta_{m, R}^{(1)}}^{\infty} R^{\prime}\left(\beta_{m, R-1}^{(1)}\right) \ldots \int_{\beta_{m, 3}^{(1)}}^{\infty} R^{\prime}\left(\beta_{m, 2}^{(1)}\right) \int_{\beta_{m, 2}^{(1)}}^{\infty} R^{\prime}\left(\beta_{m, 1}^{(1)}\right) \\
& \times d \beta_{m, 1}^{(1)} d \beta_{m, 2}^{(1)} \ldots d \beta_{m, R-1}^{(1)} d \beta_{m, R}^{(1)} .
\end{aligned}
$$

$R^{\prime}\left(\beta_{m, r}^{(1)}\right)$ is a function of only $\beta_{m, r}^{(1)}$ as $\gamma_{m}^{(1)}$ and $\Theta$ are constant within the $R$-fold integral $R\left(\beta_{m, 1}^{(1)}, \beta_{m, 2}^{(1)}, \ldots, \beta_{m, R}^{(1)}\right)$, and has the form

$$
R^{\prime}\left(\beta_{m, r}^{(1)}\right)=\exp \left(-\frac{\left(\beta_{m, r}^{(1)}\right)^{2} \gamma_{m}^{(1)}}{2 \sin ^{2}(\Theta)}\right) p\left(\beta_{m, r}^{(1)}\right) .
$$

With the assumption of the same fading parameter among the multipaths, one obtains

$$
R^{\prime \prime}(y)=\int_{y}^{\infty} R^{\prime}(x) d x \Rightarrow \frac{d R^{\prime \prime}(y)}{d y}=-R^{\prime}(y) .
$$

Insert the above relations into (64) and iteratively the $R$-fold integration can be reduced into a single integration with respect to $\beta_{m, R}^{(1)}$ such that [17]

$$
\begin{gathered}
R\left(\beta_{m, 1}^{(1)}, \beta_{m, 2}^{(1)}, \ldots, \beta_{m, R}^{(1)}\right)=\frac{1}{(R-1) !} \int_{0}^{\infty}\left[P\left(\beta_{m, R}^{(1)}\right)\right]^{L-R} \\
\times R^{\prime}\left(\beta_{m, R}^{(1)}\right)\left[R^{\prime \prime}\left(\beta_{m, R}^{(1)}\right)\right]^{R-1} d \beta_{m, R}^{(1)} .
\end{gathered}
$$

Therefore, the error probability can be written in the form of triple-level integration

$$
\begin{aligned}
P_{e}=\frac{1}{\pi} & \left(\frac{L !}{(R-1) !(L-R) !}\right)^{M} \int_{0}^{\frac{\pi}{2}} \prod_{m=1}^{M}\left[\int_{0}^{\infty}\left[P\left(\beta_{m, R}^{(1)}\right)\right]^{L-R}\right. \\
& \left.\times R^{\prime}\left(\beta_{m, R}^{(1)}\right)\left[R^{\prime \prime}\left(\beta_{m, R}^{(1)}\right)\right]^{R-1} d \beta_{m, R}^{(1)}\right] d \Theta .
\end{aligned}
$$

\section{NUMERICAL EXAMPLES}

In this section, the impacts of various parameters on the proposed system performance are illustrated. The following parameters are assumed unless explicitly specified: The number of subcarriers $M=8$, the processing gain $N=32$, the number of active users $K=20$, chip period $T_{c}=2 \mathrm{~ns}$, the rolloff factor of the square-root raised-cosine filter $\alpha=0.25$, the number of taps per side in the two-sided transversal filter $W=12$, the number of correlators per rake for each subcarrier $R=3$, and the number of paths per subband $L=10$. The decay rate $\delta$, the fading figure $\mu$, and the power ratio $v$ are taken as $10^{-1.61}, 3.5$, and $10^{-0.4}$, respectively [10]. Error probabilities of MMSE and NCC receivers are shown in most figures for performance comparison.

Fig. 4 shows the spectra of modified chip shape, corresponding to two different narrow-band spectra locations: $q_{m}=0$ and $q_{m}=0.4$. It is assumed that $J_{o} / P=40 \mathrm{~dB}$, $P / \eta_{o}=10 \mathrm{~dB}$, and $p_{m}=0.025$. It can be seen that in addition to raised cosine shaping, narrow-band notching is created. The most efficient notching appears when the narrow-band interference is located at the center frequency of the UWB subband. For $q_{m} \neq 0$, the notching is created not only in the position of narrow-band interference, but also in the symmetric location about the origin. Partial clean spectrum has to be notched. This is due to periodicity and the even function property of the frequency response of the notch filter (3). Once the impact of the 


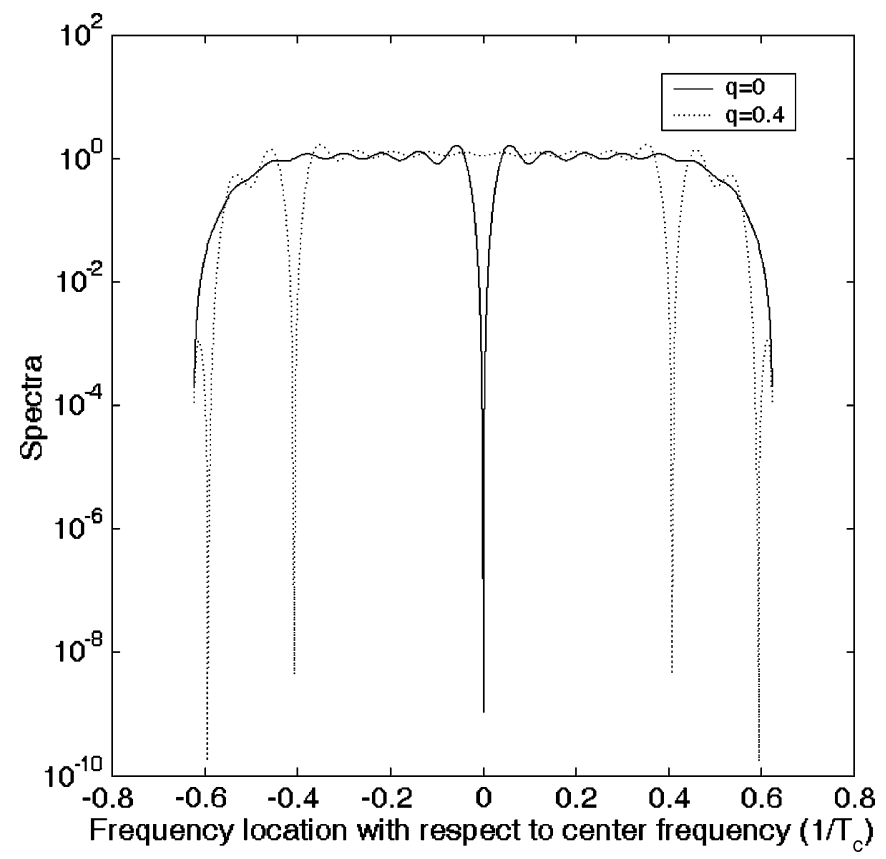

Fig. 4. Comparison of the baseband spectra of the notch filtered chip-shapes.

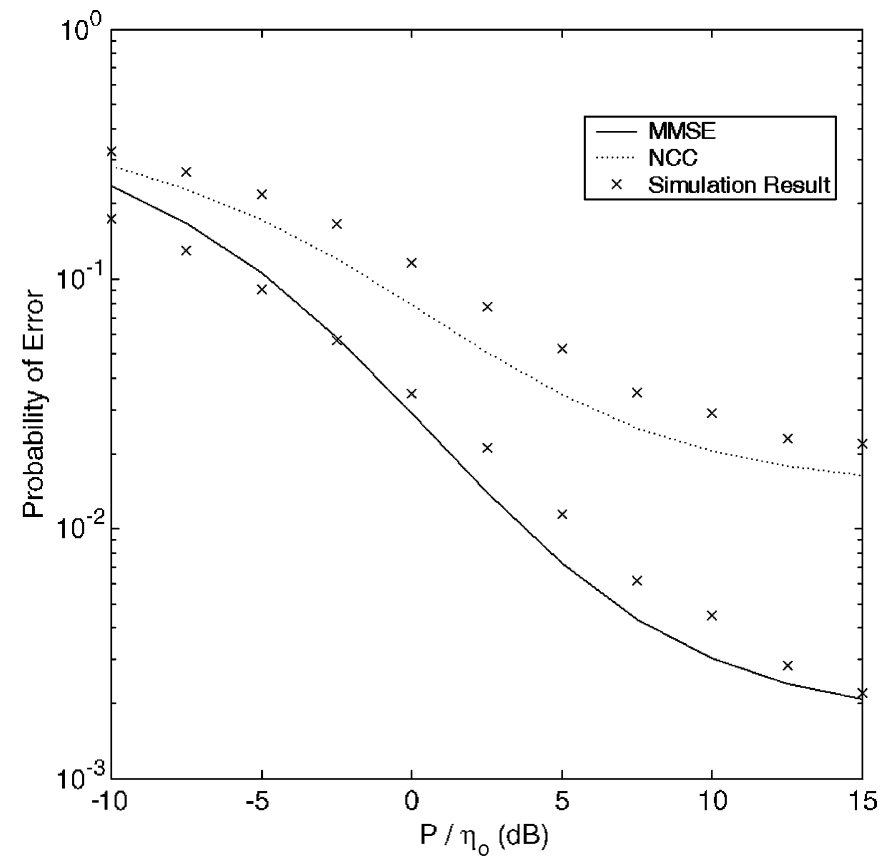

Fig. 5. Probability of error versus the signal power to noise ratio $P / \eta_{o}$.

UWB signal on the narrow-band system is reduced, then it is unnecessary to turn off the subbands overlaid on the established services since it is too inefficient for spectrum utilization.

The performance curves of two receiving strategies against $P / \eta_{o}$ are given in Fig. 5. Analytical and simulation results are obtained under these conditions: $J_{o} / P=40 \mathrm{~dB}, P / \eta_{o}=$ $10 \mathrm{~dB}, p_{m}=0.05, q_{m}=0$, and the number of narrow-band interferers $M_{i}=4$. The use of MMSE receiver can improve the overlay system performance by about one order of magnitude. It is observed that the analytical results provide a tight lower bound for the simulation ones. This is in accordance to the assumptions of random spreading code, and the adoption of

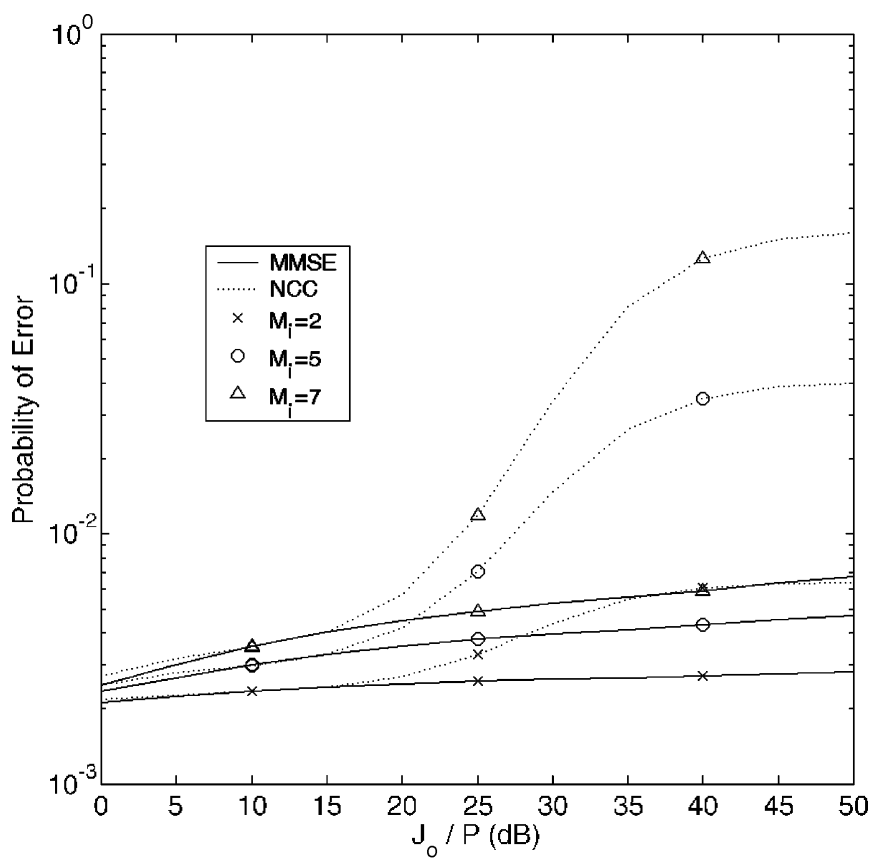

Fig. 6. Error probability as the function of jammer to signal power ratio $J_{o} / P$.

average SINR excluding the attenuation factor for all selected paths from the same subband in (59).

Fig. 6 shows error probabilities as a function of $J_{o} / P$ for different number of narrow-band interferers $\left(M_{i}=2, M_{i}=5\right.$ and $M_{i}=7$, respectively). The other system parameters are set at $P / \eta_{o}=10 \mathrm{~dB}, p_{m}=0.025$, and $q_{m}=0$. For a given number of narrow-band interferers, the performance curve of the NCC receiver has a typical S-shape representing three different phases as $J_{o} / P$ increases. The degradation is slight at first, then the error rate increases drastically and eventually the curve levels off. These observations can be accounted for by the fact that when $J_{o} / P$ is small, the notch filter at the receiver front-end provides sufficient attenuation to the narrow-band interference. However, under strong narrow-band signal, the notch introduced by the filter is too shallow for interference suppression. As $J_{o} / P$ further increases, the residual narrow-band interference continues to grow. The reliability of the decision statistic from the polluted subband decreases rapidly. Eventually, the contributions from the polluted subbands are no longer useful, which is indicated by the flattened performance curve. In contrast, for the MMSE receiver, the increase of $J_{o} / P$ only leads to a moderate growth in error probability. In comparison to the NCC receiver, the MMSE receiver is able to provide valid protection under very large $J_{o} / P$, and is robust to the number of interferers which is typical for the UWB application scenario.

Fig. 7 illustrates the error probability as a function of the number of active users $K$ for $J_{o} / P=30$ and $60 \mathrm{~dB}$, respectively. It is assumed that $p_{m}=0.05, q_{m}=0, M_{i}=3$, and $P / \eta_{o}=5 \mathrm{~dB}$. It can be seen that for a given error probability, the number of users supported by the UWB system is much affected by $J_{o} / P$ for the NCC receiver, but it is only a little dependent on $J_{o} / P$ for the MMSE receiver. That is, the MMSE receiver is more capable of suppressing strong narrow-band interference than the NCC receiver. This is consistent with the findings from Fig. 6. 


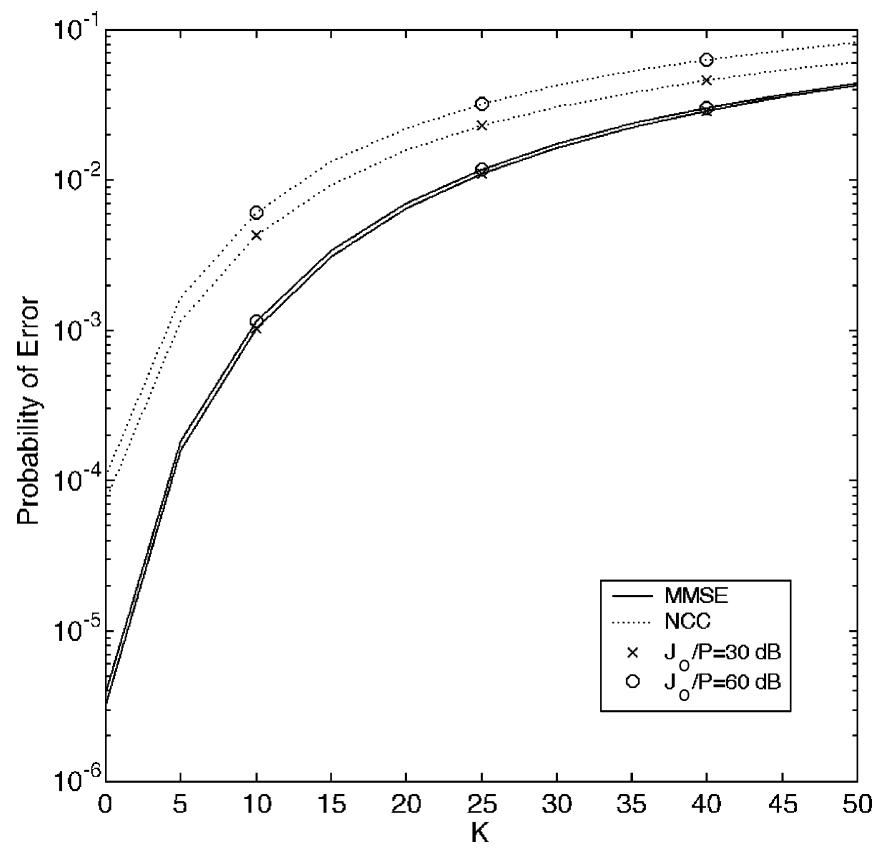

Fig. 7. BER against the number of active UWB system users $K$.

There is apparent performance difference between the MMSE receiver and NCC receiver, and this can be explained by the interference suppression and statistic combining mechanisms. For an NCC receiver, the action of NBI rejection is driven by the existence of NBI itself. It is separated from the despreading processing and the decision making. The NBI suppressions among the subbands are independent. The notch filtering process solely attempts to mitigate the bad effect of NBI but neglects other types of interferences. The benefit of frequency diversity can exercise only at the decision statistic combining. For an MMSE receiver, the despreading merges into the adaptive filtering. In addition, frequency diversity is involved in interference mitigation because the tap adaptation is based on the symbol decision feedback. The "clean" subbands not only contribute to the overall symbol decision but also enhance the correctness of the decision from the "polluted" subbands, in turn further improving the reliability of the overall symbol decision. In other words, an MMSE receiver allows effective utilization of diversity from multicarrier transmission.

It is desired to seek for a balance point between the exploration of multipath diversity and the receiver complexity. The effect of the number of rake fingers per subcarrier $R$ on the performance of the MC-CDMA system is illustrated in Fig. 8 for $P / \eta_{o}=5$ and $10 \mathrm{~dB}$, respectively. It is assumed that $J_{o} / P=$ $30 \mathrm{~dB}, p_{m}=0.05, q_{m}=0$, and $M_{i}=3$. It can be seen that when the number of correlators $R$ increases, the error probability decreases sharply at the beginning but becomes flat as $R$ approaches $L$. The marginal performance improvement diminishes for large $R$. This means that low complexity rake can achieve acceptable performance, but the additional cost would fall on the path selective circuitry.

Fig. 9 shows the error probability as a function of bandwidth ratio of narrow-band system to UWB subband $p_{m}$. The cases of $J_{o} / P=30,45$, and $60 \mathrm{~dB}$, respectively, are chosen for comparison. The remaining parameters are $P / \eta_{o}=10 \mathrm{~dB}$, $q_{m}=0$, and $M_{i}=3$. The focus is placed on the NCC re-

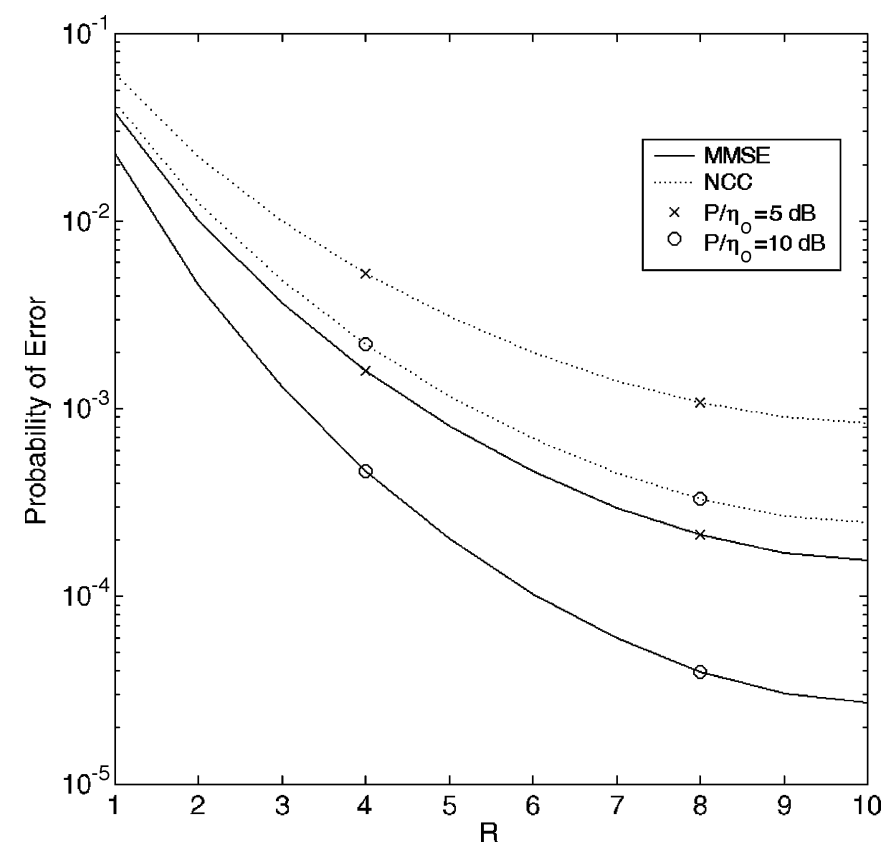

Fig. 8. Relationship between error probability and number of correlator per rake $R$.

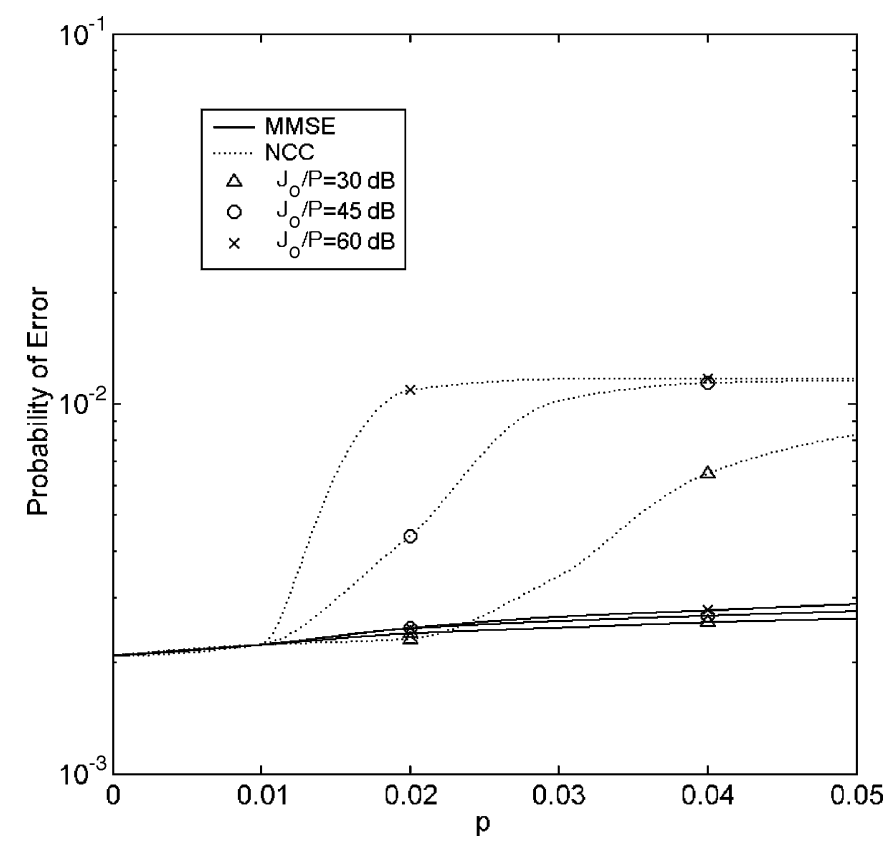

Fig. 9. Probability of error versus the bandwidth ratio $p_{m}$.

ceiver first. When $J_{o} / P=30 \mathrm{~dB}$, an NCC receiver is effective to suppress narrow-band interference, so error probability slightly increases when $p_{m}$ increases. When $J_{o} / P=45 \mathrm{~dB}$, the NCC receiver is marginally effective. Its ability to suppress narrow-band interference steadily decreases when $p_{m}$ increases. When $J_{o} / P=60 \mathrm{~dB}$, the error probability increases dramatically, and the level-off curve indicates that the symbol decision cannot rely on the contributions from the jammed subbands. However, it can be seen that for the MMSE receiver, system performance degrades slightly almost regardless of $J_{o} / P$ when $p_{m}$ increases for fixed $M_{i}$.

In Fig. 10, error probability is plotted against the frequency offset ratio of the narrow-band system from the subcarrier center when $p_{m}=0.025$. The performance curves for 


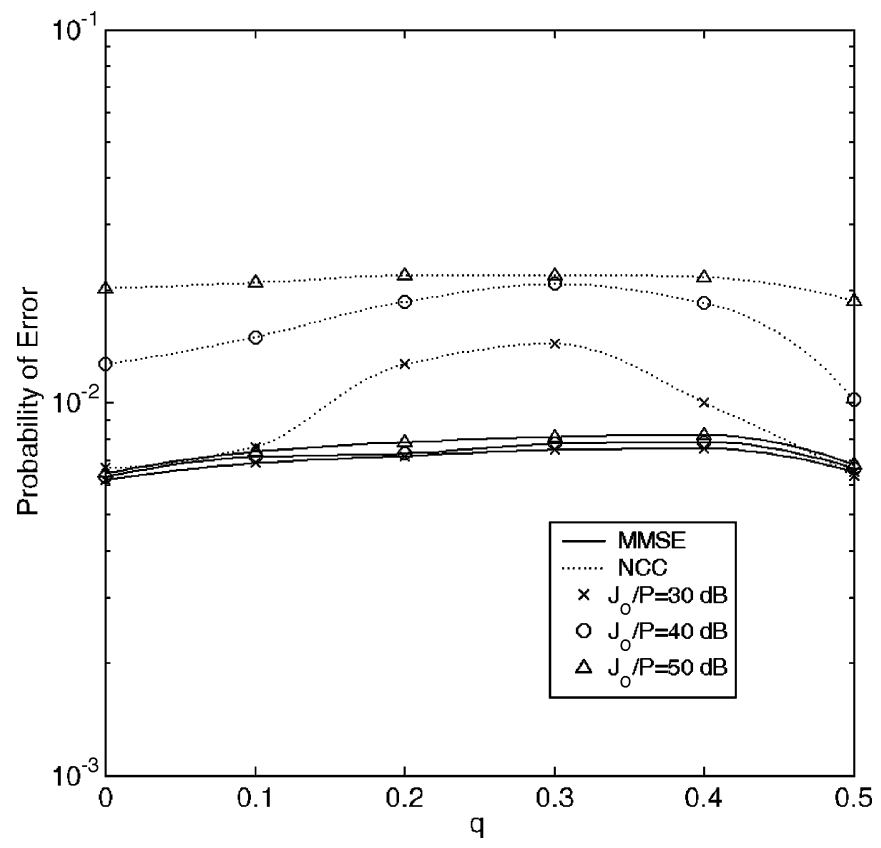

Fig. 10. System performance as the frequency offset ratio $q_{m}$ varies.

$J_{o} / P=30,, 40$, and $50 \mathrm{~dB}$ are shown. It can be seen that the MMSE receiver provides more stable performance than the NCC receiver as $q_{m}$ varies. The performance curve of NCC receiver follows the findings from Fig. 4. The error rate rises gradually at first, then drops again when the narrow-band system frequency location is moving away from the center of the subband. This is because the most efficient notch is produced when $q_{m}=0$. When $q_{m}$ takes a nonzero value, partial clean spectrum is notched. Transmitted power is reduced and error rate increases. When $q_{m}$ further increases, less transmitted power is dropped as the notch is introduced at the boundary of the spectra of the chip shape.

\section{CONCLUSIONS}

In this paper, performance expressions are obtained for the MMSE and NCC receivers for the MC-CDMA overlay in UWB applications. Performance comparisons have been made extensively between the MMSE and NCC receivers. Based on the numerical results, the MMSE receiver is robust to combat strong narrow-band interference, and to combat a large number of narrow-band interferers. In addition, the MMSE receiver is not as sensitive to the bandwidth and location of narrow-band interferers as the NCC receiver. MMSE receiver is a more suitable candidate than NCC receiver for signal detection. However, the concept of notch filtering, which is originated from NCC receiver, can be applied at a transmitter to reduce the effect of overlaying UWB signals to established services.

\section{REFERENCES}

[1] "FCC first report and order regarding ultra-wideband transmission systems,", Order (FCC 02-48), Apr. 22, 2002

[2] J. Wang and L. B. Milstein, "Multicarrier CDMA overlay for ultra-wideband communications," IEEE Trans. Commun., Oct. 2004.

[3] S. Kondo and L. B. Milstein, "Performance of multicarrier DS CDMA systems," IEEE Trans. Commun., vol. 44, pp. 238-246, Feb. 1996.

[4] B. J. Rainbolt and S. L. Miller, "The necessity for and use of CDMA transmitter filtering in overlay systems," IEEE J. Sel. Areas Commun., vol. 16, pp. 1756-1764, Dec. 1998.
[5] M. Latva-aho and M. J. Juntti, "LMMSE detection for DS-CDMA systems in fading channels," IEEE Trans. Commun., vol. 48, pp. 194-199, Feb. 2000.

[6] N. Kong and L. B. Milstein, "Average SNR of a generalized diversity selection combining scheme," IEEE Commun. Lett., vol. 3, pp. 57-59, Mar. 1999.

[7] C. R. C. M. da Silva and M. D. Yacoub, "A generalized solution for diversity combining techniques in fading channels," IEEE Trans. Microwave Theory Tech., vol. 50, pp. 46-50, Jan. 2002.

[8] L. B. Milstein, "Interference rejection techniques in spread spectrum communications," Proc. IEEE, vol. 76, pp. 657-671, Jun. 1988.

[9] J. G. Proakis, Digital Communications, 4th ed. New York: McGrawHill, 2001

[10] D. Cassioli, M. Z. Win, and A. F. Molisch, "The ultra-wide bandwidth indoor channel: from statistical model to simulations," IEEE J. Sel. Areas Commun., vol. 20, pp. 1247-1257, Aug. 2002.

[11] M. Nakagami, "The m-Distribution-a general formula of intensity distribution of rapid fading," in Statistical Methods in Radio Wave Propagation. Oxford, U.K.: Pergamon, 1960.

[12] H. V. Poor and X. Wang, "Code-aided interference suppression for DS/CDMA communications-Part I: Interference suppression capability," IEEE Trans. Commun., vol. 45, pp. 1101-1111, Sep. 1997.

[13] W. Xu and L. B. Milstein, "MMSE interference suppression for multicarrier DS-CDMA in frequency selective channels," in IEEE Global Telecommunications Conf. (GLOBECOM 98), vol. 1, 1998, pp. 259-264.

[14] A. Papoulis and S. U. Pillai, Probability, Random Variables and Stochastic Processes, 4th ed. New York: McGraw-Hill, 2002.

[15] L. S. Gradsbteyn and J. M. Ryzbik, Table of Integrals, Series, and Products, 6th ed. San Diego, CA: Academic, 2000.

[16] J. W. Craig, "A new, simple and exact result for calculating the probability of error for two-dimensional signal constellations," in Conf. Rec. IEEE 1991 Military Communications Conf. (MILCOM '91), vol. 2, 1991 , pp. $571-575$

[17] A. Annamalai and C. Tellambura, "A new approach to performance evaluation of generalized selection diversity receivers in wireless channels," in IEEE 54th Vehicular Technology Conf. (VTC 2001), vol. 4, 2001, pp. 2309-2313.

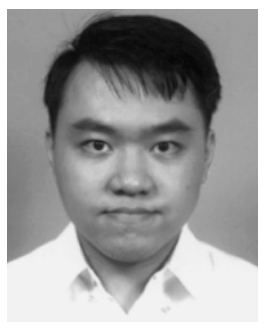

Wong Tat Tung received the B.Eng. and M.Phil. degrees from the University of Hong Kong, Hong Kong, China, in 2001 and 2003, respectively.

From 2003 to 2004, he was a Research Assistant with the University of Hong Kong. His research interests are in theory and techniques for wireless spreadspectrum communications.

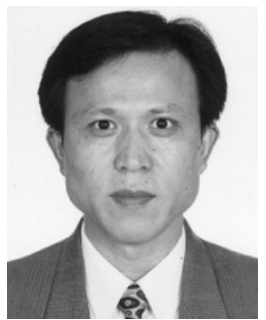

Jiangzhou Wang (M'91-SM'94) received the B.S and M.S. degrees from Xidian University, Xian, China, in 1983 and 1985, respectively, and the Ph.D. degree (with greatest distinction) from the University of Ghent, Belgium, in 1990, all in electrical engineering.

From 1990 to 1992, he was a Postdoctoral Fellow with the University of California at San Diego, here he worked on the research and development of cellular CDMA systems. From 1992 to 1995, he was a Senior System Engineer at Rockwell International Corporation, Newport Beach, CA, where he worked on the development and system design of wireless communications. Since 1995, he has been with the University of Hong Kong, China, where he is currently an Associate Professor and Coordinator of the Telecommunications Group. He was a Visiting Professor with NTT DoCoMo, Japan. Dr. Wang has published more than 100 papers in the areas of wireless mobile and spread-spectrum communications. He has written/edited two books: Broadband Wireless Communications (Norwell, MA: Kluwer, 2001) and Advances in 3G Enhanced Technologies for Wireless Communications (Reading, MA: Artech House, 2002). He has received one U.S. patent in the GSM system.

Dr. Wang is an Editor for IEEE TRANSACTIONS ON COMMUNICATIONS and a Guest Editor for IEEE JOURNAL ON SELECTED AREAS IN COMMUNICATIONS. He is listed in Who's Who in the World. He was a Technical Chairman of the 2000 IEEE Workshop in 3G Mobile Communications. He has published more than 30 IEEE TRANSACTIONS/JOURNAL papers. 